\title{
Fast algorithms for liquid crystal modelling
}

\author{
Thomas P. Bennett ${ }^{a}$, Yogesh Murugesan ${ }^{a}$, Keith R. Daly $^{b}$, Giovanni De Matteis $^{c}$ and Giampaolo \\ D'Alessandro $^{a}$ \\ ${ }^{a}$ Mathematical Sciences, University of Southampton, Southampton, England, UK; \\ ${ }^{b}$ Engineering and the Environment, University of Southampton, Southampton, England, UK \\ ${ }^{c}$ Mathematics and Information Sciences, Northumbria University, Newcastle, England, UK
}

\begin{abstract}
We review different modeling and computational methods to determine the $\mathcal{Q}$-tensor representation of the director field alignment in the absence of defects. Under this condition it is possible to represent the reorientation dynamics of the director field as the motion of the $\mathcal{Q}$-tensor over an invariant manifold. This new representation allows us to develop very accurate codes for the alignment that are orders of magnitude faster than an equivalent full $\mathcal{Q}$-tensor code. We illustrate this principle by discussing the case of a pure liquid crystal with or without flow and the case of a liquid crystal doped with fixed metallic nano-inclusions.
\end{abstract}

Keywords: Liquid crystals, Mathematical modeling of alignment, Computational methods

\section{INTRODUCTION}

Liquid crystalline materials find application in a wide range of electro-optical devices such as hybrid two beam coupling cells, ${ }^{1}$ optical wave guides, ${ }^{2}$ spatial light modulators ${ }^{3}$ and displays. ${ }^{4}$ Mathematical modeling and numerical simulations are valuable tools in optimizing these devices and understanding their operating mechanism. Traditionally, there are two approaches to model liquid crystal alignment. In the Oseen-Frank formalism ${ }_{i}^{5}$ the director field is represented with a unit vector $\hat{\boldsymbol{n}}$, while the Landau de Gennes framework ${ }^{6}$ represent the director field using a $3 \times 3$ traceless, symmetric tensor, the $\mathcal{Q}$-tensor.

From a computational point of view the two approaches have both pros and cons. The vector representation of the director gives rise to equations with only one time constant that are, hence, computationally efficient. On the other hand, this formalism suffers from the intrinsic ambiguity that $\hat{\boldsymbol{n}}$ and $-\hat{\boldsymbol{n}}$ represent the same director alignment. The $\mathcal{Q}$-tensor representation, instead, is generic and can represent without any ambiguity any director alignment. It also embodies the nematic symmetry automatically. Moreover, this theory takes into account the orientational order of the liquid crystal by including the thermotropic energy in addition to the elastic counterpart. On the other hand, due to the two energy contributions, the corresponding dynamical equations for the director alignment have two considerably different time scales and the resulting computational model is, hence, very stiff and hard to compute efficiently. In this paper, we use the $\mathcal{Q}$ representation of the liquid crystal alignment and present computationally efficient numerical approximations that exploit the difference in scales between different contributions to the free energy away from defects to model the following three systems of relevance to liquid crystal based electro-optical devices: (a) a pure nematic liquid crystal; (b) a pure nematic liquid crystal that flows; (c) a nematic liquid crystal with fixed inclusions.

A salient feature of these systems is the existence of an invariant manifold defined by the thermotropic free energy which dominates the free energy of the system away from the defects. The elastic, electrostatic, and flow effects act as perturbations that drive the dynamics of the liquid crystal along this manifold. By defining the invariant manifold based on the dominant thermotropic energy and rewriting the dynamic equations constrained to the manifold a set of non-stiff equations are obtained. In the absence of defects these equations are accurate, computationally efficient and capture all the relevant features of the microscale dynamics through a set of effective macroscopic parameters. These models are ideal for solving liquid crystal alignment in electro-optical devices that often do not have topological defects in the bulk in order to minimize scattering losses and are doped with nanoparticles to improve their response to the applied electro-magnetic fields.

Corresponding author: G. D’Alessandro (dales@ soton.ac.uk) 
Section 2, presents the approximation to compute the liquid crystal alignment far away from defect in pure nematic liquid crystals. This approximation is named the Defect Free $\mathcal{Q}$-tensor approximation (DFQTA); section 3 and 4, extends this approximation to include the effects of fluid flow and systems doped with fixed inclusions respectively.

\section{DEFECT FREE $\mathcal{Q}$-TENSOR APPROXIMATION (DFQTA) FOR PURE LIQUID CRYSTALS}

In this section, the $\mathcal{Q}$-tensor that describes the alignment of liquid crystals is introduced and the free energy of the liquid crystal and the equations that describe the dynamics of liquid crystal are presented. The concept of invariant manifold and DFQTA equations that describe this manifold and dynamics of liquid crystal alignment on the manifold are given. Computation of alignment in a typical liquid crystal cell using this approximation is also presented to illustrate the application of the model. Full details of the mathematical derivation of the equations can be found in Daly et al. ${ }^{7}$

\subsection{Modeling nematic liquid crystals}

The tensorial order parameter $\tilde{\mathcal{Q}}$ that quantifies the structure of a nematic liquid crystalline phase with scalar order parameter $\tilde{S}$ and biaxial order parameter $\tilde{\beta}$ is given by

$$
\tilde{\mathcal{Q}}=\sqrt{\frac{3}{2}} \tilde{S}(\hat{\boldsymbol{n}} \otimes \hat{\boldsymbol{n}})^{[S]}+\sqrt{\frac{3}{2}} \tilde{\beta}(\hat{\boldsymbol{m}} \otimes \hat{\boldsymbol{m}})^{[S]} .
$$

This normalized $\tilde{\mathcal{Q}}$ is symmetric and traceless and $\operatorname{Tr}\left(\tilde{\mathcal{Q}}^{2}\right)=\tilde{S}^{2}$ for $\tilde{\beta}=0$. The unit directors $\hat{\boldsymbol{n}}$ and $\hat{\boldsymbol{m}}$ represents major and minor axis along which the liquid crystal molecules are aligned. The superscript $[S]$ denotes a symmetric and traceless tensor. A generic $3 \times 3$ tensor $\mathcal{N}$ can be made symmetric and traceless by

$$
\left(\mathcal{N}_{i j}\right)^{[S]}=\frac{\mathcal{N}_{i j}+\mathcal{N}_{j i}}{2}-\frac{1}{3} \mathcal{N}_{k k} \delta_{i j}
$$

where $\delta_{i j}$ is the Kronecker delta.

The steady state alignment of a nematic liquid crystal is the solution of the Euler-Lagrange equation for the minimum of the free energy. A generic free energy, $\tilde{\mathcal{F}}$, of a nematic liquid crystal is of the form

$$
\tilde{\mathcal{F}}=\tilde{\mathcal{F}}_{t}(\tilde{\mathcal{Q}})+\tilde{\mathcal{F}}_{d}(\tilde{\mathcal{Q}}, \tilde{\nabla} \tilde{\mathcal{Q}})+\tilde{\mathcal{F}}_{e}(\tilde{\mathcal{Q}}, \tilde{\mathcal{E}})
$$

where $\tilde{\mathcal{F}}_{t}, \tilde{\mathcal{F}}_{d}$ and $\tilde{\mathcal{F}}_{e}$ are, respectively, the thermotropic, elastic, and electrostatic free energies. $\tilde{\mathcal{E}}$ is the symmetric and traceless electric field tensor defined by

$$
\tilde{\mathcal{E}}=\sqrt{\frac{3}{2}}(\tilde{\boldsymbol{E}} \otimes \tilde{\boldsymbol{E}})^{[S]}
$$

Here $\tilde{\boldsymbol{E}}$ is the electric field, given by $\tilde{\boldsymbol{E}}=-\tilde{\boldsymbol{\nabla}} \tilde{\varphi}$, where $\tilde{\varphi}$ is the electric potential.

In order to minimize the algebra, in this paper we assume that the free energy costs for splay, twist and bend deformations of the director field are all equal (single elastic constant approximation). However, it is possible to apply the methodology outlined in this paper also to liquid crystal with different elastic constants. Therefore, we write the free energy (3) as

$$
\tilde{\mathcal{F}}=\frac{\tilde{A}}{2}\left(\tilde{T}-\tilde{T}^{*}\right) \operatorname{Tr}\left(\tilde{\mathcal{Q}}^{2}\right)-\sqrt{6} \tilde{B} \operatorname{Tr}\left(\tilde{\mathcal{Q}}^{3}\right)+\frac{\tilde{C}}{2} \operatorname{Tr}^{2}\left(\tilde{\mathcal{Q}}^{2}\right)+\frac{\tilde{L}_{1}}{2}|\tilde{\nabla} \tilde{\mathcal{Q}}|^{2}-\tilde{\chi}_{a} \operatorname{Tr}(\tilde{\mathcal{Q}} \tilde{\mathcal{E}}),
$$

where the first three terms represent the thermotropic energy and the last two the elastic and the electrostatic energy, respectively. In this expression $\tilde{T}$ is the temperature of the bulk of the liquid crystal; $\tilde{T}^{*}$ is the pseudo-critical temperature, i.e. the temperature at which the isotropic phase becomes unstable; $\tilde{A}, \tilde{B}$ and $\tilde{C}$ are the bulk thermotropic coefficients independent of temperature; $\tilde{L}_{1}$ is defined as $\tilde{L}_{1}=\tilde{K} /\left(3 \tilde{S}^{2}\right)$ where $\tilde{K}$ is the single elastic constant of the liquid crystal material; $\tilde{\chi}_{a}$ is the dielectric anisotropy coefficient. 
To ensure that the traceless and symmetric properties of $\tilde{\mathcal{Q}}$ and $\tilde{\mathcal{E}}$ are respected, we follow the approach of Sonnet $e t$ $a l .{ }^{8}$ and express $\tilde{\mathcal{Q}}$ and $\tilde{\mathcal{E}}$ in terms of their components on the basis of the traceless $3 \times 3$ symmetric tensors,

$$
\tilde{\mathcal{Q}}=\sum_{p=1}^{5} \tilde{a}_{p} T^{(p)} \quad \text { and } \quad \tilde{\mathcal{E}}=\sum_{p=1}^{5} \tilde{e}_{p} T^{(p)},
$$

where

$$
\begin{array}{rlrl}
T^{(1)} & =\frac{1}{\sqrt{6}}\left(-\hat{\boldsymbol{e}}_{1} \otimes \hat{\boldsymbol{e}}_{1}-\hat{\boldsymbol{e}}_{2} \otimes \hat{\boldsymbol{e}}_{2}+2 \hat{\boldsymbol{e}}_{3} \otimes \hat{\boldsymbol{e}}_{3}\right), & \\
T^{(2)} & =\frac{1}{\sqrt{2}}\left(\hat{\boldsymbol{e}}_{1} \otimes \hat{\boldsymbol{e}}_{1}-\hat{\boldsymbol{e}}_{2} \otimes \hat{\boldsymbol{e}}_{2}\right), & T^{(3)} & =\frac{1}{\sqrt{2}}\left(\hat{\boldsymbol{e}}_{1} \otimes \hat{\boldsymbol{e}}_{2}+\hat{\boldsymbol{e}}_{2} \otimes \hat{\boldsymbol{e}}_{1}\right), \\
T^{(4)} & =\frac{1}{\sqrt{2}}\left(\hat{\boldsymbol{e}}_{1} \otimes \hat{\boldsymbol{e}}_{3}+\hat{\boldsymbol{e}}_{3} \otimes \hat{\boldsymbol{e}}_{1}\right), & T^{(5)}=\frac{1}{\sqrt{2}}\left(\hat{\boldsymbol{e}}_{2} \otimes \hat{\boldsymbol{e}}_{3}+\hat{\boldsymbol{e}}_{3} \otimes \hat{\boldsymbol{e}}_{2}\right),
\end{array}
$$

where $\hat{\boldsymbol{e}}_{k}$ for $k=1,2,3$ are the Cartesian basis vectors, and $\tilde{a}_{p}$ and $\tilde{e}_{p}$ are the $p$-th components of the vectors $\tilde{\boldsymbol{a}}, \tilde{\boldsymbol{e}}$ respectively.

To facilitate the comparison of the magnitude of the various contributions to the free energy, we non-dimensionalise the free energy given in eq. (5) with the following set of scaling relations

$$
\begin{array}{ll}
(x, z)=(\tilde{x}, \tilde{z}) / \tilde{L} & \text { Scaled lengths; } \tilde{L} \text { is the typical cell length } \\
F=\frac{81 \tilde{C}^{3}}{16 \tilde{B}^{4}} \tilde{F} & \text { Scaled free energy } \\
\mathcal{Q}=\frac{3 \tilde{C}}{2 \tilde{Q}} \tilde{\mathcal{Q}} & \text { Scaled alignment tensor } \\
\boldsymbol{a}=\frac{3 \tilde{C}}{2 \tilde{B}} \tilde{\boldsymbol{a}} & \text { Scaled alignment tensor components } \\
\mathcal{E}=\tilde{\mathcal{E}}\left(\frac{\tilde{L}}{\tilde{\varphi}_{0}}\right)^{2} & \text { Scaled electric field tensor, } \tilde{\varphi}_{0} \text { typical voltage } \\
\boldsymbol{e}=\tilde{\boldsymbol{e}}\left(\frac{\tilde{L}}{\tilde{\varphi}_{0}}\right)^{2} & \\
\tilde{T}_{c}-\tilde{T}^{*}=\frac{2 \tilde{B}^{2}}{9 \tilde{A} \tilde{C}}, & \text { Scaled electric field tensor components } \\
T_{0}=\frac{1}{2} \frac{\tilde{T}_{c}-\tilde{T}^{*}}{\tilde{T}_{c}-\tilde{T}^{*}}, & \text { Scaled temperature } \\
\xi_{0}^{2}=\frac{9 \tilde{K}^{*} \tilde{C}}{2 \tilde{B}^{2} \tilde{L}^{2}}, & \text { Non-dimensional elastic constant } \\
\chi_{a}=\tilde{\chi}_{a} \frac{27 \tilde{\varphi}_{0}^{2} \tilde{C}^{2}}{2 \tilde{L}^{2} \tilde{B}^{3}}, & \text { The dielectric anisotropy coefficient } \\
t=\frac{\tilde{t}}{\tilde{\tau}_{0}} & \text { Scaled time } \\
\tilde{\tau}_{0}=\frac{9 \tilde{C}}{4 \tilde{B}^{2}} \tilde{\zeta}_{1}, & \text { Time constant for scaling time; } \tilde{\zeta}_{1} \text { is the rotational viscosity }
\end{array}
$$

The dimensionless free energy in terms of $a$ and $e$ is

$$
\mathcal{F}=\underbrace{\frac{T_{0}}{2}|\boldsymbol{a}|^{2}-\sqrt{6} \sum_{p, q, r} \operatorname{Tr}\left(T^{(p)} T^{(q)} T^{(r)}\right) a_{p} a_{q} a_{r}+\frac{1}{2}|\boldsymbol{a}|^{4}}_{\mathcal{F}_{t}}+\underbrace{\frac{\xi_{0}^{2}}{2}|\boldsymbol{\nabla} \boldsymbol{a}|^{2}}_{\mathcal{F}_{d}}-\underbrace{\chi_{a} \boldsymbol{a} \cdot \boldsymbol{e}}_{\mathcal{F}_{e}}
$$




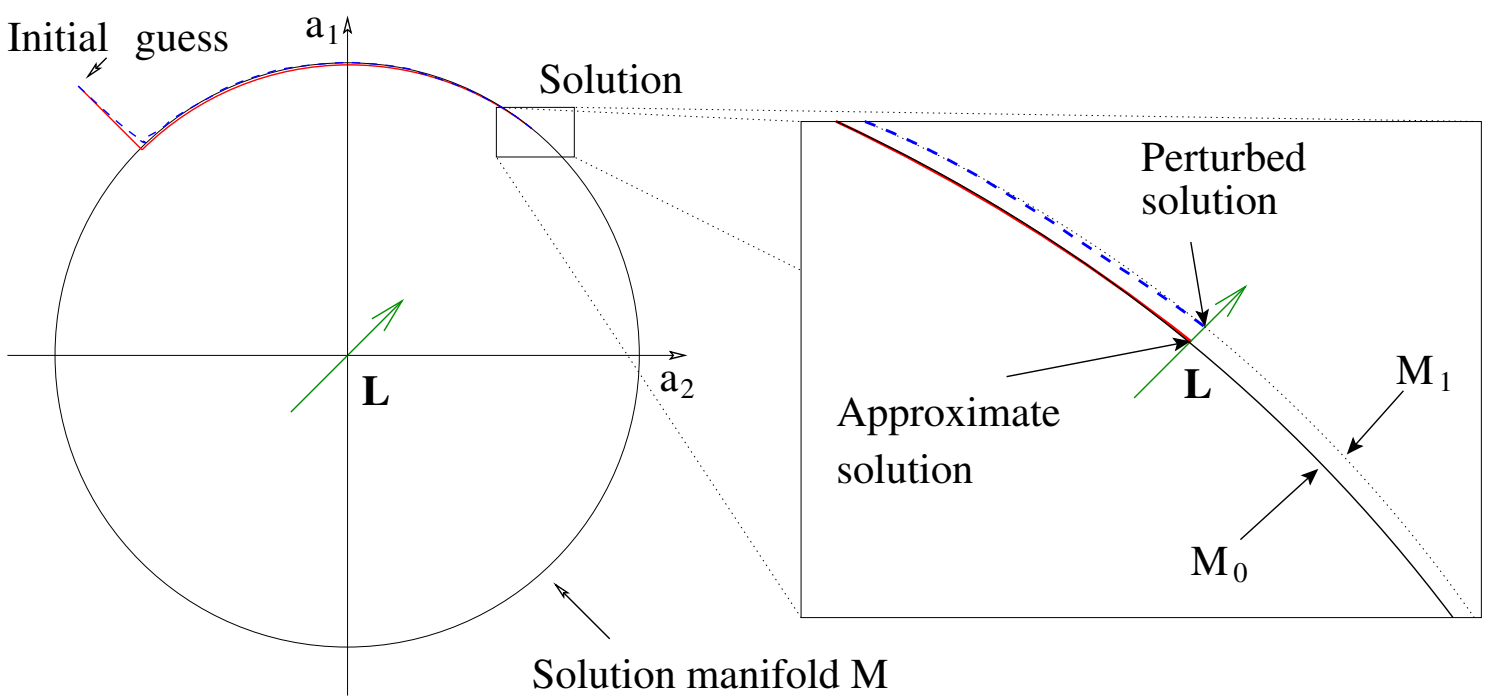

Figure 1. Graphical representation of the invariant manifold that corresponds to the minimum of the thermotropic free energy (circle), of the fast motion towards the manifold (straight red radial segment) and of the slow reorientation motion along the manifold (red circular trajectory). The dashed line represents a numerical solution of eq. (10). [From Daly et al. ${ }^{7}$ ]

where $a_{p}$ and $e_{p}$ are the components of $\boldsymbol{a}$ and $\boldsymbol{e}$ respectively, $T_{0}$ is the scaled temperature, $\xi_{0}^{2}$ is the scaled elastic constant, and $\chi_{a}$ is the scaled dielectric anisotropy coefficient. The corresponding equation of motion describing the dynamics of liquid crystal alignment is given by Euler-Lagrange equations which minimize eq. (9):

$$
\xi_{0}^{2} \nabla^{2} \boldsymbol{a}+\chi_{a} \boldsymbol{e}+M(\boldsymbol{a}) \boldsymbol{a}=\frac{\partial \boldsymbol{a}}{\partial t},
$$

where $t$ is the scaled time, see eq. (8). $M$ is the thermotropic matrix given by

$$
M(\boldsymbol{a})=T_{0} \mathcal{I}+\left[\begin{array}{ccccc}
3 a_{1} & -3 a_{2} & -3 a_{3} & \frac{3}{2} a_{4} & \frac{3}{2} a_{5} \\
-3 a_{2} & -3 a_{1} & 0 & \frac{3}{2} \sqrt{3} a_{4} & -\frac{3}{2} \sqrt{3} a_{5} \\
-3 a_{3} & 0 & 3 a_{1} & \frac{3}{2} \sqrt{3} a_{5} & \frac{3}{2} \sqrt{3} a_{4} \\
\frac{3}{2} a_{4} & \frac{3}{2} \sqrt{3} a_{4} & \frac{3}{2} \sqrt{3} a_{5} & \frac{3}{2} a_{1}+\frac{3}{2} \sqrt{3} a_{2} & \frac{3}{2} \sqrt{3} a_{3} \\
\frac{3}{2} a_{5} & -\frac{3}{2} \sqrt{3} a_{5} & \frac{3}{2} \sqrt{3} a_{4} & \frac{3}{2} \sqrt{3} a_{3} & \frac{3}{2} a_{1}-\frac{3}{2} \sqrt{3} a_{2}
\end{array}\right],
$$

and $\mathcal{I}$ is the identity matrix.

For length scales of typical devices $T_{0} \sim O(1)$, while $\xi_{0}^{2} \sim O\left(10^{-7}\right)$ and $\chi_{a} \sim O\left(10^{-6}\right)$. Therefore, away from defects, where $\nabla^{2} \boldsymbol{a} \simeq O\left(1 / \xi_{0}^{2}\right)$, the term $M(\boldsymbol{a}) \boldsymbol{a}$ in eq. (10) is $O(1)$ while the other two are $O\left(10^{-6}\right)$. In other words, the large difference in magnitude of the thermotropic part of the free energy with respect to the elastic and electrostatic parts results in the appearance of two vastly different time scales in the dynamic alignment eq. (10), which make it stiff and hard to integrate numerically. A variety of techniques such as adaptive meshing and director renormalization has been attempted to address this issue. ${ }^{9}$ In this work, we demonstrate the existence of an invariant manifold in these systems and develop an approximation that harnesses the separation of scale to obtain equations that describe the reorientation dynamics of liquid crystals away from the defects. This is achieved by treating the elastic and electrostatic free energy contributions as a perturbation to the thermotropic free energy, resulting in equations of motion that are non-stiff. At the core of the defect, $\nabla^{2} \boldsymbol{a} \simeq O\left(1 / \xi_{0}^{2}\right)$ and $\mathcal{F}_{d}$ is of the same order as that of $\mathcal{F}_{t}$. In this case the approximation does not hold any more and one must resort to integrating the full $\mathcal{Q}$-tensor eq. (10).

\subsection{A simple example of invariant manifold}

In order to demonstrate the concept of invariant manifold, we present the simpler case of a $2 \times 2 \mathcal{Q}$-tensor: this represents a nematic director confined to a plane. Its vector representation $\boldsymbol{a}$ has only two components, $a_{1}$ and $a_{2}$, so that it is easy to 
visualize the evolution of the $\mathcal{Q}$-tensor in the $\left(a_{1}, a_{2}\right)$-plane, as shown in figure 1 . The equations of motions consist of a rotationally symmetric thermotropic part $M(\boldsymbol{a}) \boldsymbol{a}=\left[T_{0}+2|\boldsymbol{a}|^{2}\right] \boldsymbol{a}$, perturbed by small symmetry breaking contributions from the elastic and electrostatic energies, $\mathcal{L}(\boldsymbol{a}, \boldsymbol{e}, \boldsymbol{\nabla} \boldsymbol{a})=\xi_{0}^{2} \nabla^{2} \boldsymbol{a}+\chi_{a} \boldsymbol{e}$. As the thermotropic term $M(\boldsymbol{a}) \boldsymbol{a}$ is $\operatorname{SO}(2)$ invariant its critical points form a circle $M_{0}$, with equation $a_{1}^{2}+a_{2}^{2}=-T_{0} / 2$, in the two dimensional $\left(a_{1}, a_{2}\right)$-plane (see figure 1). This is an invariant manifold for the dynamics of the liquid crystal, if we exclude the effect of the elastic and electrostatic energies. From a generic initial condition, the dynamics rapidly converges on a fast timescale to the manifold $M_{0}$ (red radial segment in figure 1) and in the absence of elastic or electrostatic forces, moves no further.

The effect of the perturbation $\mathcal{L}$ is to break the symmetry and to collapse the invariant manifold to two critical points (one stable and one unstable). The system evolves on a slow time scale towards the stable one of these. If the perturbation is small then the orbit lies very close to the invariant manifold $M_{0}$. This is the case illustrated in figure 1 , where we can see that the numerical solution (dashed blue line) remains very close to the equilibrium circle $M_{0}$.

This observation forms the basis of the approximation scheme summarized in the next section. We assume that the system is at equilibrium with respect to its thermotropic part, i.e. $M(\boldsymbol{a}) \boldsymbol{a}=0$, so that the $\mathcal{Q}$-tensor lies on the invariant manifold $M_{0}$ (the circle in figure 1). We then restrict the time derivative of the $\mathcal{Q}$-tensor components, $\dot{\boldsymbol{a}}$, to be tangent to the manifold, so that the reorientation dynamics of the $\mathcal{Q}$-tensor is described by an orbit on $M_{0}$ (circular red arch in figure 1 ). Therefore, the configuration of the liquid crystal is described by a set of algebraic equations to identify the manifold and a set of differential equations for the slow motion tangent to it. The key advantage is that the dynamical equations have only one time scale and can therefore be integrated numerically very efficiently.

\subsection{The DFQTA equations}

The ideas outlined in the previous section can be applied to the full $\mathcal{Q}$-tensor eq. (10) to obtain alignment equations for a $3 \times 3 \mathcal{Q}$-tensor in the absence of defects.

We take $\eta=\xi_{0}^{2}$ as a small parameter and expand the components of $\boldsymbol{a}$ as a power series $a_{n}=a_{n, 0}+\eta a_{n, 1}+O\left(\eta^{2}\right)$, $n=1, \ldots, 5$. We also introduce fast, $t_{0}=t$, and a slow, $t_{1}=\eta t$, time variables so that $\partial_{t}=\partial_{t_{0}}+\eta \partial_{t_{1}}$ in eq. (10). We assume that the system is at equilibrium on the fast time scale, i.e. it is on the invariant manifold, and set $\partial_{t_{0}} a_{n, q}=0$, so that we can write the three dimensional Euler-Lagrange eq. (10) that describes the dynamics of the liquid crystal as

$$
\eta\left(\nabla^{2} \boldsymbol{a}+\chi_{a} \boldsymbol{e}\right)+M(\boldsymbol{a}) \boldsymbol{a}=\eta \frac{\partial \boldsymbol{a}}{\partial t_{1}} .
$$

where $\chi_{0}=\chi_{a} / \xi_{0}^{2}$.

Formally, the procedure outlined in the previous section consists in expanding this equations in powers of $\eta$, collecting terms at each order and applying the required solvability conditions. ${ }^{7}$ The end result is a set of two equations that describe the 3-manifold in the five-component space and a set of three differential equations that describe the reorientation dynamics tangent to the manifold. The manifold equations are

$$
\begin{aligned}
& S_{0}^{2} \equiv \sum_{p=1}^{5} a_{p, 0}^{2}=\left[\frac{3+\sqrt{\left(9-8 T_{0}\right)}}{4}\right], \\
& S_{0}^{3}=a_{1,0}^{3}+3 a_{1,0}^{2}\left(S_{0}-a_{1,0}\right)+\frac{3 \sqrt{3}}{2}\left[a_{2,0}\left(a_{4,0}^{2}-a_{5,0}^{2}\right)+2 a_{3,0} a_{4,0} a_{5,0}\right] .
\end{aligned}
$$

The first equation fixes the value on the manifold of the leading order term in the power series expansion of the scalar order parameter, $S=S_{0}+O(\eta)$. The second equation states that on the invariant manifold the leading order term of the biaxial order parameter expansion, $\beta=\beta_{0}+O(\eta)$ is zero.

The time dependent equations of motion on the manifold are

$$
\boldsymbol{V}^{(n)} \cdot \frac{\partial \boldsymbol{a}_{0}}{\partial t_{1}}=\boldsymbol{V}^{(n)} \cdot\left(\nabla^{2} \boldsymbol{a}_{0}+\chi_{a} \boldsymbol{e}\right), \quad n=1,2,3,
$$



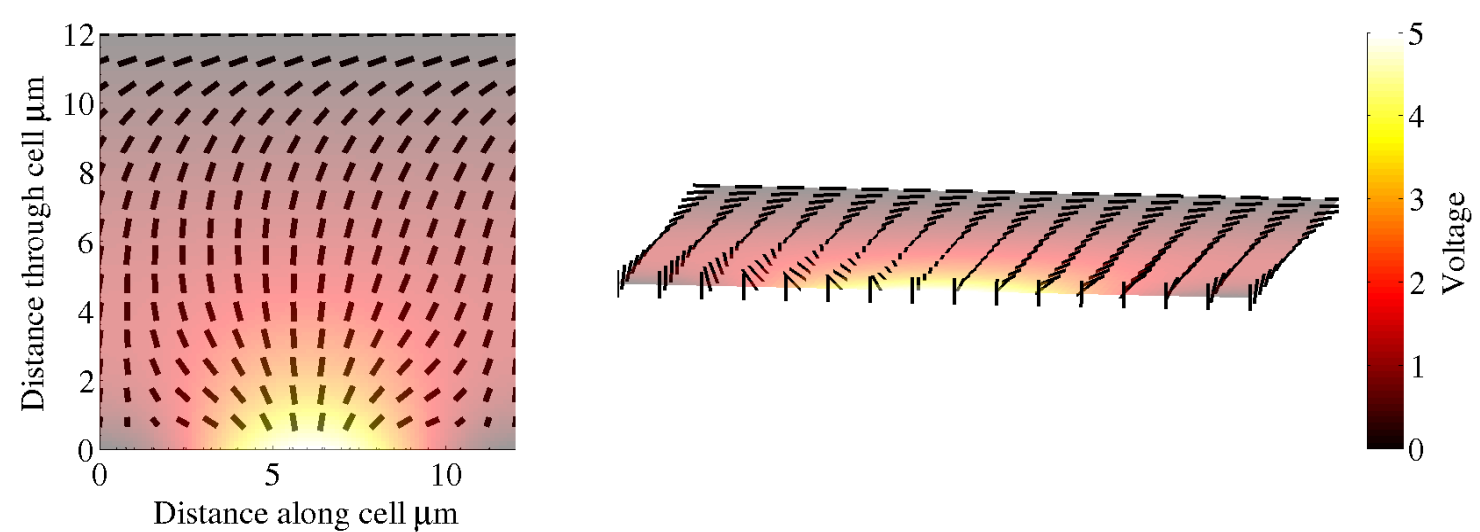

Figure 2. The 3D director plots for a twist cell for a 5 Volt spatially modulated electric field computed using the DFQTA equations (14). The shading and the color bar corresponds to the voltage through out the cell measured in Volts.

where $\boldsymbol{a}=\boldsymbol{a}_{0}+\eta \boldsymbol{a}_{1}+O\left(\eta^{2}\right)$ and $\boldsymbol{V}^{(n)}$ are three vectors that span the space tangent to the manifold at $\boldsymbol{a}_{0}$. In terms of the components of the alignment vector $\boldsymbol{a}_{0}$ they are

$$
\boldsymbol{V}^{(1)}=\left(\begin{array}{c}
0 \\
-2 a_{3,0} \\
2 a_{2,0} \\
-2 a_{5,0} \\
2 a_{4,0}
\end{array}\right), \quad \boldsymbol{V}^{(2)}=\left(\begin{array}{c}
-\sqrt{3} a_{4,0} \\
a_{4,0} \\
a_{5,0} \\
\sqrt{3} a_{1,0}-a_{2,0} \\
-a_{3,0}
\end{array}\right), \quad \boldsymbol{V}^{(3)}=\left(\begin{array}{c}
\sqrt{3} a_{5,0} \\
a_{5,0} \\
-a_{4,0} \\
a_{3,0} \\
-\sqrt{3} a_{1,0}-a_{2,0}
\end{array}\right)
$$

Equations (14) are the end result of the DFQTA: they describe the reorientation dynamics of the liquid crystal on the defectfree manifold. They are non-stiff and can be easily solved on a desktop PC even for complex geometries. Computing the director alignment using this approximation uses a fraction of the computation resources required to solve the stiff full $\mathcal{Q}$-tensor equations (10): two order of magnitude speed gains can be easily achieved. ${ }^{7}$

We demonstrate the method by solving the DFQTA equations (14) for a twist cell, an interesting device that allows for three dimensional orientation of the liquid crystals. The test geometry is a square in the $(x, z)$-plane. Periodic boundary conditions are imposed in the $x$-direction and Dirichlet boundary conditions corresponding to strong planar anchoring are imposed at $z=0$ and $z=L_{z}$. The liquid crystal director lie in the $(x, z)$-plane at $z=L_{z}$ and perpendicular to it at $z=0$. Figure 2 shows the computed $3 \mathrm{D}$ director profile for a 5 Volt spatially modulated electric field. The MATLAB codes that solves the DFQTA equations using spectral methods used to compute the alignment and generate the director plots presented in the figure can be downloaded from the following link: http://www.personal.soton.ac.uk/dales/DFQTA/.

\section{EXTENDING THE DFQTA TO INCLUDE FLOW}

In liquid crystal devices, switching between different states is usually achieved by applying or removing an electric field. In order to understand the physics underlying their switching mechanism and kinetics, incorporating the effect of flow of liquid crystalline material is essential. One approach to numerically simulating the switching dynamics of these devices is to obtain the alignment and fluid flow equations from the most generic dissipation function that satisfies the symmetries of the system. In this section we explore how it is possible to extend the defect free $\mathcal{Q}$ approximation developed in section 2 to include the hydrodynamics of the liquid crystal. By constraining the temporal evolution of the alignment of liquid crystals on to the invariant manifold, the resulting equations are non-stiff. A first validation of the model has been the successful verification that in the limit of a planar cell, the approximations reduce to dynamic equations in terms of director angle $\theta$ derived by Clark and Leslie. ${ }^{10}$ Further validations and the numerical integration of the resulting equations are in progress. Here we explain why the DFQTA can be applied to the $\mathcal{Q}$-tensor equations with flow. The derivation of the equations and their complete verification is deferred to a future publication. ${ }^{11}$ 


\subsection{Dynamic equations}

The dissipation function formulated by Sonnet $e t a i^{12}$ has been used to derive the coupled dynamics of flow and alignment of a uniaxial nematic liquid crystal. As in the case of the DFQTA, it is essential to write the dissipation function in nondimensional variables in order to identify correctly the relative size of each term. The dissipation function and the dynamics equations are made non-dimensional by using the following set of scaling laws in addtion to those in eq. (8):

$$
\begin{array}{ll}
R=\tilde{R} \frac{\tilde{\tau}_{0}^{3}}{\tilde{L}^{2} \tilde{\rho}}, & \text { Non-dimensional dissipation function, } \tilde{\rho} \text { is the density } \\
\tilde{v}_{0}=\frac{\tilde{L}}{\tilde{\tau}_{0}}, & \text { Typical speed, used as velocity scaling coefficient } \\
\boldsymbol{v}=\frac{\tilde{\boldsymbol{v}}}{\tilde{v}_{0}}, & \text { Non-dimensional velocity } \\
\Sigma_{k}=\frac{\tilde{\zeta}_{k} \tilde{\tau}_{0}}{\tilde{L}^{2} \tilde{\rho}}\left(\frac{2 \tilde{B}}{3 \tilde{C}}\right)^{\alpha_{k}}, & \text { Non-dimensional viscosities for flow equation, } k=\{1, \ldots, 8\} \\
\mathcal{T}=\tilde{\mathcal{T}} \frac{\tilde{\tau}_{0}^{2}}{\tilde{L}^{2} \tilde{\rho}}, & \text { Non-dimensional stress tensor } \\
p=\frac{\tilde{p}}{\tilde{p}_{0}}, & \text { Reference pressure } \\
\tilde{p}_{0}=\frac{\tilde{L}^{2} \tilde{\rho}}{\tilde{\tau}_{0}^{2}}, & \text { Scaling constant for elastic contribution to flow equation } \\
K=\frac{16 \tilde{B}^{4} \tilde{\tau}_{0}^{2}}{81 \tilde{C}^{3} \tilde{L}^{2} \tilde{\rho}}, & \text { Non-dimensional viscosities for alignment equation, } k=\{2,3\} \\
\zeta_{k}=\frac{\Sigma_{k}}{\Sigma_{1}}, &
\end{array}
$$

Here the coefficients $\tilde{\zeta}_{k}, k=1, \ldots, 8$ are dimensional viscosity coefficients defined in Sonnet et $a_{i}^{12}$ and the exponent $\alpha_{k}$ is 0 for $k=8,1$ for $k=\{2,4\}$ and 2 otherwise. The non-dimensionalised dissipation function $R$ that satisfies the symmetries of a uniaxially aligned liquid crystal is ${ }^{12}$

$$
\begin{aligned}
R= & \frac{1}{3} \Sigma_{1} \operatorname{Tr}\left(\stackrel{\mathcal{Q}}{ }^{2}\right)+\sqrt{\frac{2}{3}} \Sigma_{2} \operatorname{Tr}(\mathcal{D} \mathcal{Q})+\frac{2}{3} \Sigma_{3} \operatorname{Tr}(\mathcal{D} \mathcal{Q} \mathcal{Q})+\frac{1}{\sqrt{6}} \Sigma_{4} \operatorname{Tr}\left(\mathcal{D}^{2} \mathcal{Q}\right)+ \\
& \frac{1}{3} \Sigma_{5} \operatorname{Tr}\left(\mathcal{D}^{2} \mathcal{Q}^{2}\right)+\frac{1}{3} \Sigma_{6}[\operatorname{Tr}(\mathcal{D} \mathcal{Q})]^{2}+\frac{1}{3} \Sigma_{7} \operatorname{Tr}\left(\mathcal{Q}^{2}\right) \operatorname{Tr}\left(\mathcal{Q}^{2}\right)+\frac{1}{2} \Sigma_{8} \operatorname{Tr}\left(\mathcal{D}^{2}\right)
\end{aligned}
$$

Here $\mathcal{Q}$ is the co-rotational derivative of $\mathcal{Q}$, defined as

$$
\stackrel{\mathcal{Q}}{=}=\partial_{t} \mathcal{Q}+\boldsymbol{v} \cdot \nabla \mathcal{Q}-[\mathcal{W}, \mathcal{Q}],
$$

where $[\mathcal{W}, \mathcal{D}]$ is the commutator of $\mathcal{W}$ and $\mathcal{D}$ who are the anti-symmetric and symmetric parts of the velocity gradient tensor, given by

$$
\mathcal{W}=\frac{1}{2}\left[(\boldsymbol{\nabla} \boldsymbol{v})-(\boldsymbol{\nabla} \boldsymbol{v})^{T}\right] \quad \text { and } \quad \mathcal{D}=\frac{1}{2}\left[(\boldsymbol{\nabla} \boldsymbol{v})+(\boldsymbol{\nabla} \boldsymbol{v})^{T}\right] .
$$

In liquid crystal systems with dynamic coupling between flow and alignment, the non-dimensional governing equation for the flow is

$$
\dot{\boldsymbol{v}}=\frac{\partial \boldsymbol{v}}{\partial t}+(\boldsymbol{v} \cdot \nabla) \boldsymbol{v}=\nabla \cdot \mathcal{T},
$$


where $\mathcal{T}$ is the dimensionless stress tensor, given by

$$
\begin{aligned}
& \mathcal{T}=-p \mathcal{I}-K \xi_{0}^{2}(\nabla \mathcal{Q} \odot \nabla \mathcal{Q}) \\
& +\sqrt{\frac{2}{3}} \Sigma_{2} \mathcal{Q}+\frac{2}{3} \Sigma_{3}(\mathcal{Q} \mathcal{Q})^{[S]}+\sqrt{\frac{2}{3}} \Sigma_{4}(\mathcal{D} \mathcal{Q})^{[S]}+\frac{2}{3} \Sigma_{5}\left(\mathcal{D} \mathcal{Q}^{2}\right)^{[S]} \\
& +\frac{2}{3} \Sigma_{6} \operatorname{Tr}(\mathcal{D} \mathcal{Q}) \mathcal{Q}+\frac{2}{3} \Sigma_{7} \operatorname{Tr}\left(\mathcal{Q}^{2}\right) \mathcal{D}+\Sigma_{8} \mathcal{D} \\
& +\frac{2}{3} \Sigma_{1}(\mathcal{Q} \mathcal{Q}-\mathcal{Q} \mathcal{Q})+\sqrt{\frac{2}{3}} \Sigma_{2}(\mathcal{Q D}-\mathcal{D} \mathcal{Q})+\frac{2}{3} \Sigma_{3}\left[\mathcal{Q}(\mathcal{D} \mathcal{Q})^{[S]}-(\mathcal{D} \mathcal{Q})^{[S]} \mathcal{Q}\right] .
\end{aligned}
$$

The symbol $(\nabla \mathcal{Q} \odot \nabla \mathcal{Q})$ is defined in Cartesian components as ${ }^{12}(\nabla \mathcal{Q} \odot \nabla \mathcal{Q})_{p q}=\mathcal{Q}_{i j, p} \mathcal{Q}_{i j, q}$. These equations are coupled to the dimensionless alignment equation:

$$
\frac{2}{3} \mathcal{Q}+\sqrt{\frac{2}{3}} \zeta_{2} \mathcal{D}+\frac{2}{3} \zeta_{3}(\mathcal{D} \mathcal{Q})^{[S]}-\xi_{0}^{2} \nabla^{2} \mathcal{Q}-\chi_{a} \mathcal{E}+T_{0} \mathcal{Q}-\frac{3 \sqrt{6}}{2}\left(\mathcal{Q}^{2}\right)^{[S]}+\operatorname{Tr}\left(\mathcal{Q}^{2}\right) \mathcal{Q}=0
$$

These governing equations along with the incompressibility condition $\boldsymbol{\nabla} \cdot \boldsymbol{v}=0$ gives the sets of equations to be solved to obtain the dynamics of flow and alignment of liquid crystal. Eq. (22) is the equivalent of eq. (10) for the case without flow expressed in $\mathcal{Q}$-tensor notation, rather than in component form.

\subsection{DFQTAF approximation}

Under the assumption that the flow reaches equilibrium instantaneously, we can set the right hand side of eq. (20) to zero. In this case, the magnitude of the flow terms in eq. (22) is $\sim O\left(10^{-7}\right)$. Following the invariant manifold technique demonstrated for pure nematic liquid crystal in the absence of flow which display similar separation of scales between different contribution to the free energy, flow effects can also be treated as small perturbation along with elastic and electrostatic contributions as demonstrated in section 2 . We have already obtained a preliminary version of the defect free $\mathcal{Q}$ tensor approximation with flow (DFQTAF) equations, that can compute the coupled dynamics of flow and alignment of liquid crystals. They have been validated by verifying that in the limit of a planar cell, dynamic equations derived from phenomenological model by Clark and Leslie. ${ }^{10}$ Further validation of the dynamic equations and their numerical integration are under way and will be presented in a separate future publication. ${ }^{11}$

\section{LIQUID CRYSTALS DOPED WITH METALLIC NANOPARTICLES}

There is considerable research interest in colloidal, or doped, nematic liquid crystals. These consist of micron to nanometer sized particles suspended in a nematic host. Dopants may posses permanent electric ${ }^{1314}$ or magnetic dipoles ${ }^{15 \cdots 19}$ leading to ferronematic systems, or their influence may be due to mechanical anchoring on the dopant surface that can lead to remarkable defect structures. ${ }^{20}{ }^{21}$ Here we focus on metallic, e.g. gold, nanoparticles. ${ }^{22}$ In these cases the liquid crystal alignment is dominated by elastic and electrostatic forces with the dopants acting to alter rather than dominate the properties of the liquid crystal. Their effect, however, can be significan ${ }^{23 \cdot 25}$ and may lead to applications related to plasmon tuning in self-assembled structures ${ }^{26.27}$ or tunable metamaterials. ${ }^{28}$

The existence of an invariant manifold for the $\mathcal{Q}$-tensor equations in the absence of defects can also be used to model in a computationally efficient way nematic liquid crystals doped with nanoparticles. Our group has derivec ${ }^{29}$ equations describing the long range alignment of a nematic liquid crystal hosting periodically arranged rigid metallic inclusions of arbitrary shape and fixed orientation. The liquid crystal is assumed to obey weak anchoring on the surface of the inclusions. These may be identified with rigid non-rotating dopant particles or with a periodic micro-structure. We are interested in the case where the anchoring of the nematic to the inclusions is sufficiently weak as to not induce defects. Instead, the inclusions alter the elastic and electrostatic response of the nematic. A schematic of the system studied is shown in figure 3 . the domain of the system is composed of unit cells of size $L_{y}$ and the macroscopic size of the system is $L_{x}$, with $L_{y} \ll L_{x}$. Treating the full system numerically is computationally expensive due to the fine mesh needed to resolve the microstructure and the stiffness of the governing equations. ${ }^{7}$ Rather than solving the Euler-Lagrange equations for the alignment of the nematic in the complete domain pictured in figure 3 we have derived a set of computationally efficient equations for the macroscopic description of this system. ${ }^{29}$ In this case approximate equations are obtained using homogenization 


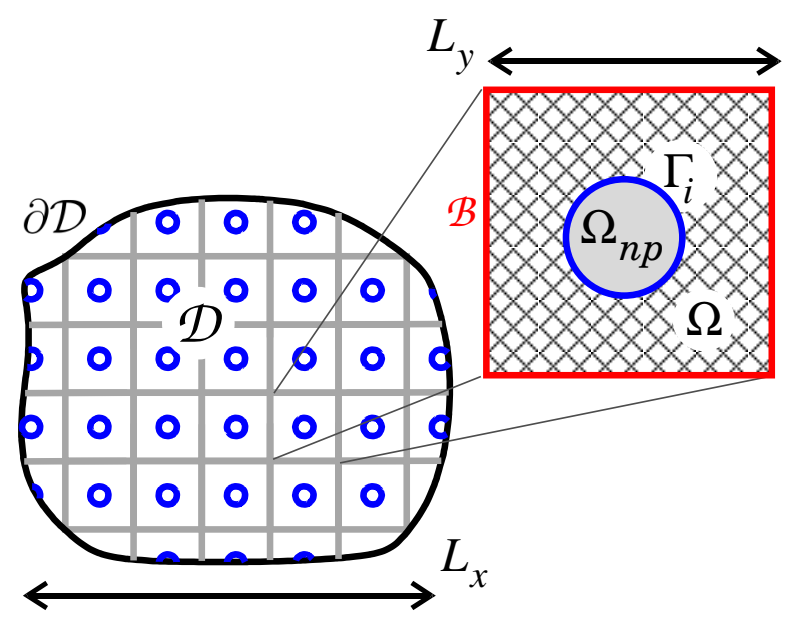

Figure 3. Domains used in defining scale separation taken from Bennett et al. ${ }^{29}$ The macroscopic domain consists of an open region $\mathcal{D}$ containing nematic liquid crystal, with external boundary $\partial \mathcal{D}$, constructed from unit cells $\Omega$ with outer boundaries $\mathcal{B}$ and metallic inclusions of volume $\Omega_{n p}$ and boundary $\Gamma_{i}$, with $i$ an index that runs over the total number of inclusions. We seek a description of the nematic in the limit as $L_{y}$ tends to zero with $L_{x}$ fixed.

theory ${ }^{30}$ rather than a multiple scale expansion as in the DFQTA approximation, but both asymptotic methods rely on the existence of the manifold to yield non-stiff dynamical equations for the reorientation of the liquid crystals. The detailed calculations for the derivation of the approximate director equations are available in Bennett et al. ${ }^{29} \mathrm{Here}$, as in the previous sections, we summarize the methods and list the main results. In section 4.1 we outline the system studied, in section 4.2 we present and interpret the model, referring for the full details to Bennett et al. ${ }^{29}$ In section 4.3 we present an example applications of the approximate macroscopic equations.

\subsection{Modeling the doped system}

The liquid crystal orientation is described by means of a free energy minimization over the volume of the nematic $\mathcal{D}$, and boundary with the inclusions $\left(\cup_{i} \Gamma_{i}\right)$, with $\Gamma_{i}$ the boundary of the $i$-th particle, subject to Dirichlet conditions on the macroscopic boundary $\partial \mathcal{D}$ (see figure 3). The electric fields is governed by Maxwell's equation $\boldsymbol{\nabla} \cdot \boldsymbol{D}=0$ subject to floating potential boundary conditions on the metallic inclusions. Using the notation developed in section 2 we can write the (non-dimensional) governing equations for the alignment field as

$$
\begin{array}{ll}
\xi_{0}^{2} \nabla^{2} \boldsymbol{a}+\chi_{a} \boldsymbol{e}+M(\boldsymbol{a}) \boldsymbol{a}=\frac{\partial \boldsymbol{a}}{\partial t} & \boldsymbol{x} \in \mathcal{D}, \\
\hat{\boldsymbol{n}}_{S} \cdot \boldsymbol{\nabla} \boldsymbol{a}=W\left(\boldsymbol{a}_{S}-\boldsymbol{a}\right) & \boldsymbol{x} \in \Gamma, \\
\boldsymbol{a}=\boldsymbol{b}(\boldsymbol{x}) & \boldsymbol{x} \in \partial \mathcal{D},
\end{array}
$$

while those for the electric field are

$$
\begin{array}{ll}
\boldsymbol{\nabla} \cdot[(\mathcal{I}+\alpha \mathcal{Q}) \nabla \phi]=0 & \boldsymbol{x} \in \mathcal{D}, \\
\phi=V_{0}(\boldsymbol{x}) & \boldsymbol{x} \in \partial \mathcal{D}, \\
\phi=\mathrm{C}_{i} & \boldsymbol{x} \in \Gamma_{i}, \\
\int_{\Gamma_{i}} \hat{\boldsymbol{n}}_{S} \cdot(\mathcal{I}+\alpha \mathcal{Q}) \nabla \phi d S=0 & \boldsymbol{x} \in \Gamma_{i},
\end{array}
$$

with $\boldsymbol{a}, \boldsymbol{e}, \xi_{0}^{2}$ and $\chi_{a}$ defined in eq. (8) and $M(\boldsymbol{a})$ defined in (10). The prescribed value of $\boldsymbol{a}$ on the macroscopic boundary $\partial \mathcal{D}$ is given by $\boldsymbol{b}(\boldsymbol{x}) . \boldsymbol{a}_{S}$ is the preferred alignment direction on the inclusions, $\hat{\boldsymbol{n}}_{S}$ is the unit vector normal to the surface of the particle and $W=\frac{\tilde{\mu} \tilde{L}_{x}}{2 \tilde{L}_{1}}$ is the dimensionless anchoring strength, with $\tilde{\mu}$ the dimensional anchoring energy density and $\tilde{L}_{x}$ the dimensional macroscopic length scale, related to the non-dimensional macroscopic scale $L_{x}$ by $L_{x}=\tilde{L}_{x} / \tilde{L}$, with 
$\tilde{L}$ the typical cell-length defined in eq. (8). $\alpha=\sqrt{\frac{2}{3}} \frac{\Delta \epsilon}{\epsilon_{u}} \frac{2 B}{3 C}$ is the dimensionless dielectric coefficient, with $\epsilon_{u}$ and $\Delta \epsilon$ the isotropic and anisotropic dielectric coefficients respectively. $C_{i}$ is the constant potential attained on the surface $\Gamma_{i}$ of the $i$-the inclusion. It is determined by the macroscopic boundary conditions (24b). $V_{0}(\boldsymbol{x})$ is the potential on the macroscopic boundary $\partial \mathcal{D}$.

The system pictured in figure 3 contains two distinct and disparate length-scales, the macroscopic scale $L_{x}$ and microscopic $L_{y}$. These define a smallness parameter $\eta_{h}=\frac{L_{y}}{L_{x}}$. We are interested in the behavior of the system as $\eta_{h}$ tends to zero in the case where the ratio of surface to volume free energy remains constant. As we take this limit, the two phase system, particles and liquid crystals, is replaced by a single homogeneous phase, an "effective" liquid crystal. The end result is a set of "homogenized" equations that describe the macroscopic behavior of the homogeneous phase. The homogenized equations define the long-range alignment behavior of the nematic and contain effective material parameters that are related to the inclusions geometry by the "cell problems": these are sets of coupled differential equations that are defined in a single unit cell, of size $L_{y}$. We may interpret the cell problems as quantifying the changes in the nematic response to driving forces as a result of the metallic inclusions. For our system the driving forces are the elasticity of the nematic and the externally applied electrostatic field. We therefore obtain two sets of cell problems: one relates the change in elastic response of the system to the microscopic geometry; the second determines the altered dielectric response of the effective liquid crystal due to the presence of the metallic inclusions.

\subsection{Homogenization}

The alignment of the nematic can be computed by simultaneously solving equations (23) and (24) inside the full microscopically detailed geometry. To determine the cell problems and homogenized equations we define a microscopic scale $\boldsymbol{y}$ by the relation $\boldsymbol{y}=\boldsymbol{x} / \eta_{h}$ and assume that all fields are, a priori functions of $\boldsymbol{x}$ and $\boldsymbol{y}$. We use the chain rule to expand gradient operators and all variables as series in $\eta_{h}$ of the form:

$$
\begin{aligned}
\boldsymbol{\nabla} & =\frac{1}{\eta_{h}} \boldsymbol{\nabla}_{\boldsymbol{y}}+\boldsymbol{\nabla}_{\boldsymbol{x}}, \\
\boldsymbol{a} & =\boldsymbol{a}_{0}+\eta_{h} \boldsymbol{a}_{1}+\eta_{h}^{2} \boldsymbol{a}_{2}+O\left(\eta_{h}^{3}\right), \\
\phi & =\phi_{0}+\eta_{h} \phi_{1}+\eta_{h}^{3} \phi_{3}+O\left(\eta_{h}^{3}\right)
\end{aligned}
$$

We make the following observations: as observed in section $2, \xi_{0}^{2}$ and $\chi_{a}$ are small quantities. Here we assume that they are both $\mathcal{O}\left(\eta_{h}^{2}\right)$ indicating that the liquid crystal dynamics, eq. (23a), has two different time scales. ${ }^{7}$ We note that this scaling is the same as the one used in section 2 once we identify $\eta_{h}^{2}=\eta$. To maintain a constant ratio of surface to volume free energy as $\eta_{h}$ tends to zero, the anchoring energy $W$ must scale as $\eta_{h}$ : we therefore write it as $W=\eta_{h} W_{1}$, with $W_{1}$ a quantity $O(1)$. Lastly, we are only interested in the reorientation of the liquid crystal due to the elastic and electrostatic energies: as in section 2 we introduce a slow time, $t_{2}=\eta_{h}^{2} t$, and expand the time derivative as

$$
\frac{\partial}{\partial t}=\eta_{h}^{2} \frac{\partial}{\partial t_{2}} .
$$

In writing this expansion we have implicitly assumed that all faster processes have reached equilibrium. In fact, due to the identity $\eta_{h}^{2}=\eta$, the slow variable $t_{2}$ defined here is the same as the slow variable $t_{1}$ defined in section 2 . In section 2 we showed how these scalings, in the absence of inclusions, imply that the liquid crystal dynamics is confined to the invariant manifold $M_{0}$. Here we find that the homogenized equations include the additional effect of the inclusions into the drift along the invariant manifold. By substituting the expanded fields (25) into the governing equations (23) and (24) and solving at each order in $\eta_{h}$ we obtain the following results. The homogenized equations are

$$
\begin{gathered}
\boldsymbol{V}^{(k)} \cdot\left\{\xi_{0}^{2} \boldsymbol{\nabla}_{\boldsymbol{x}} \cdot\left(D \boldsymbol{\nabla}_{\boldsymbol{x}} \boldsymbol{a}_{0}\right)+\chi_{a}\left[\boldsymbol{e}_{M}\left(1+2 \frac{\left|\Omega_{n p}\right|}{|\Omega|}\right)+\boldsymbol{p}\right]+W_{1} \xi_{0}^{2} \boldsymbol{q}\right\}=\boldsymbol{V}^{(k)} \cdot \frac{\partial \boldsymbol{a}_{0}}{\partial t_{2}}, \\
\boldsymbol{\nabla}_{\boldsymbol{x}} \cdot\left[\left(|\Omega|+\left|\Omega_{n p}\right|\right)\left(\mathcal{I}+\alpha \mathcal{Q}_{0}\right) \boldsymbol{\nabla}_{\boldsymbol{x}} \phi_{0}\right]=0 .
\end{gathered}
$$

for $k=1,2,3$ where $\boldsymbol{V}^{(k)}$ are given by (15). These equations are completed by equations (13), i.e. the conditions that define the invariant manifold $M_{0}$ : the scalar order parameter is a constant determined by the temperature and the biaxiality is zero. 
The effective fields and material parameters that appear in equations (27) are given by

$$
\begin{gathered}
D_{i j}=\frac{1}{|\Omega|} \int_{\Omega}\left(\delta_{i j}+\frac{\partial \chi_{j}}{\partial y_{i}}\right) d^{3} y, \\
\boldsymbol{q}=\frac{1}{|\Omega|} \int_{\Gamma} \boldsymbol{a}_{S} d S \\
e_{M i}=\operatorname{Tr}\left[\boldsymbol{\nabla}_{\boldsymbol{x}} \phi_{0} \otimes \boldsymbol{\nabla}_{\boldsymbol{x}} \phi_{0} T^{(i)}\right], \\
p_{i}=\frac{1}{|\Omega|} \int_{\Omega} \operatorname{Tr}\left[\left(\sum_{k=1}^{3} \boldsymbol{\nabla}_{\boldsymbol{y}} R_{k} \frac{\partial \phi_{0}}{\partial x_{k}}\right) \otimes\left(\sum_{k=1}^{3} \boldsymbol{\nabla}_{\boldsymbol{y}} R_{k} \frac{\partial \phi_{0}}{\partial x_{k}}\right) T^{(i)}\right] d V .
\end{gathered}
$$

Here $T^{(i)}$ for $i=1, . ., 5$ are the basis of $3 \times 3$ traceless symmetric tensors, see eq. (7), $|\Omega|$ is the volume of a unit cell filled with liquid crystal and $\left|\Omega_{n p}\right|$ is the volume occupied by an inclusion. The fields $\chi_{k}(\boldsymbol{y})$ and $R_{k}(\boldsymbol{y}), k=1,2,3$, that appear in equations (28) and (31), respectively are the solutions of two sets of cell problems. The first set relates the change in elastic response of the system to the microscopic geometry:

$$
\begin{array}{ll}
\xi_{0}^{2} \nabla_{\boldsymbol{y}}^{2} \chi_{k}=0, & \boldsymbol{y} \in \Omega, \\
\hat{\boldsymbol{n}}_{S} \cdot \nabla_{\boldsymbol{y}} \chi_{k}=-\hat{\boldsymbol{n}}_{S} \cdot \boldsymbol{e}_{k}, & \boldsymbol{y} \in \Gamma_{i},
\end{array}
$$

where $\hat{\boldsymbol{e}}_{k}$ for $k=1,2,3$ are the Cartesian basis vectors. The second determines the altered dielectric response of the effective liquid crystal due to the presence of the metallic inclusions:

$$
\begin{array}{ll}
{\left[\delta_{i j}+\alpha \mathcal{Q}_{0 i j}\right] \frac{\partial^{2} R_{k}}{\partial y_{i} \partial y_{j}}=0} & \boldsymbol{y} \in \Omega, \\
R_{k}=-y_{k} & \boldsymbol{y} \in \Gamma_{i}, \\
\int_{\Gamma_{i}}\left(\mathcal{I}+\alpha \mathcal{Q}_{0}\right) \nabla_{\boldsymbol{y}} R_{k}(\boldsymbol{y}) \cdot d \boldsymbol{S}=0 & \boldsymbol{y} \in \Gamma_{i} .
\end{array}
$$

The material parameters and effective fields defined by equations (28)-(31) have a physical interpretation. $D_{i j}$ is the effective elasticity tensor and encodes the anisotropic excluded volume effect, $\boldsymbol{q}$ is a forcing term that favors a nematic aligned with the average preferred orientation on the inclusions. The macroscopic electric field $\boldsymbol{e}_{M}$ is amplified by the factor $\left(1+2 \frac{\left|\Omega_{n p}\right|}{|\Omega|}\right)$. This factor quantifies how much larger the drop in potential across the nematic is due to the constraint that the potential remains constant on the metallic inclusions. Finally the polarization field $\boldsymbol{p}$ results from the fringe electric fields around an inclusion: the electric field must be orthogonal to the surface of the inclusions. Hence, even a uniform external electric field will have components along all the coordinate axes close to the inclusions.

When supplemented with macroscopic boundary conditions on $\partial \mathcal{D}$ equations (27.33) form a complete macroscopic description of the liquid crystal alignment. As the DFQTA equations derived in section 2 , they are accurate and extremely fast to integrate numerically in comparison to the unapproximated equations (23 24). In Bennett $e t a i^{29}$ they have been validated by comparing their predictions with numerical simulations of the full system (23 24), for two cases of inclusions in a twisted cell. The director obeys planar anchoring on the boundaries of the cell with easy axis on the two glass plates at an angle of $\pi / 2$ radians to each other. The two cases treated are spherical dopant particles at non-zero voltage and ellipsoidal particles with no applied field. In each case homeotropic anchoring on the nanoparticles is assumed. figure 4 shows the good agreement found in both cases. The ellipsoidal particles break the symmetry of the ground state and induce a static deformation of the director even in the absence of an applied electric field. Spherical particles act to enhance the response of the nematic to the applied field as shown in top left panel of figure 4. In all the cases illustrated in this figure the numerical integration of the homogenized equations was more than 1,000 times faster than that of the full $\mathcal{Q}$-tensor equations. 

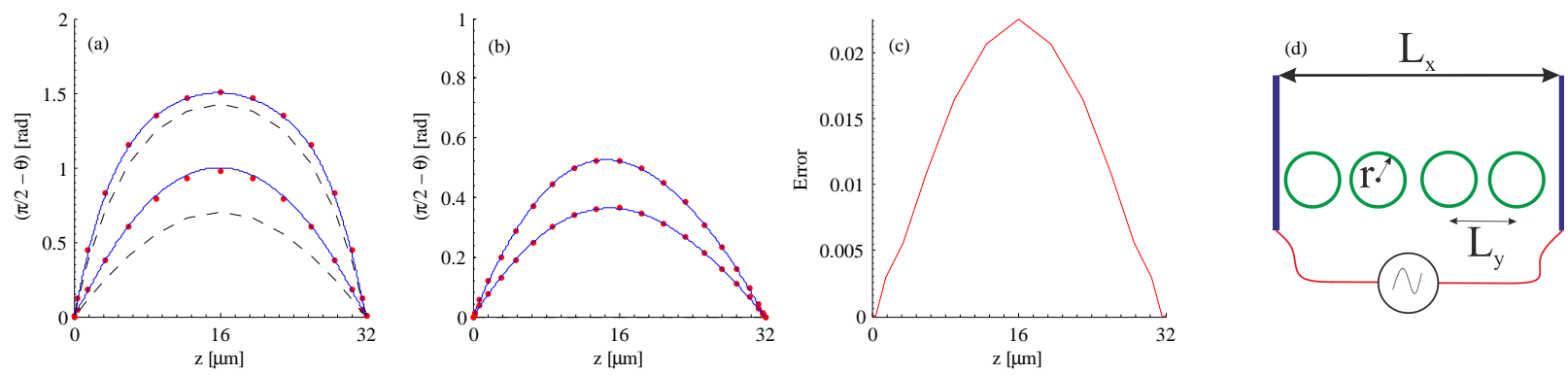

Figure 4. [Adapted from Bennett et $a_{i}{ }^{29}$ ] A comparison of full numerical simulations of the system to the solution given by the homogenized equations for spherical and ellipsoidal particles inside a $32 \mu \mathrm{m}$ twisted cell, zero pretilt and twist at boundaries and homeotropic anchoring on dopants. (a) Spherical particles of radius $r=0.3 \mu \mathrm{m}$ at applied voltages of $1.5 \mathrm{~V}$ and $3 \mathrm{~V}$ (lower and upper curves respectively). (b) Ellipsoidal dopants $V=0$ with anchoring energy $W_{1}=50$ (bottom) and $W_{1}=100$ (top) orientation given by $(\tilde{\theta}, \tilde{\phi})=\left(45^{\circ}, 30^{\circ}\right)$ with $\tilde{\theta}$ azimuthal and $\tilde{\phi}$ polar angles. The semi major axis of the ellipsoids is $0.3 \mu \mathrm{m}$ the semi minor axes are $0.1 \mu \mathrm{m}$. (a) \& (b) Red points are from homogenization, broken black line is undoped and blue line from Comsol numerical simulations. (c) Absolute error Error $=\left|\theta_{H}-\theta_{N}\right|$ where $\theta_{H}$ is the tilt angle from the homogenized equations and $\theta_{N}$ is extracted from numerical simulations of the full system. (d) Schematic diagram of the system studied: planar cell of size $L_{x}$ with spherical/ellipsoidal dopants with spacing $L_{y}$.

\subsection{Doped liquid crystals in a splay geometry}

The homogenized equations (27.33) are a very powerful tool not just to find quickly the alignment of a doped liquid crystal, but also to study the physics of devices based on these materials. In Bennett $e t a i^{29}$ the case of a splay geometry with homeotropic anchoring on the particles is discussed in some detail. The main results is that the effective elasticity tensor always reduces the elastic constant of the nematic while the metallic inclusions increase the effective field felt by the nematic. For spherical particles these effects always lower the Frederiks threshold and switch on time while increasing the switch off time. In this case the forcing term $\boldsymbol{q}$ is zero and the elasticity tensor $D_{i j}$ is a multiple of the identity. The polarisation $\boldsymbol{p}$ is non-zero: $p_{1}$ increases rapidly with particle radius while $p_{2}$ is always small, and zero if appropriate symmetry conditions are satisfied. The situation is more interesting if we consider anisotropic particles: for these $\boldsymbol{q}$ is nonzero and both $\boldsymbol{q}$ and the polarization $\boldsymbol{p}$ depend on the orientation of the particles. Careful choice of particle geometry ${ }^{29}$ can decrease both the switch on and off times and either increase or decrease the Frederiks threshold with respect to a pure liquid crystal.

To conclude this section and illustrate the genericity of the method, we consider the case of a toroidal nanoparticle whose axis lies in the $(x, z)$-plane, with $z$ the direction into the cell and $x$ the direction of the planar alignment of the liquid crystal on the cell walls. We show in figure 5 the numerical solution (obtained using Comsol) of the cell problem (32) for $\chi_{3}$. The color shading shows how the corrections to the alignment induced by the particle follow its geometrical symmetry. In figure 6 we plot, instead, some of the parameters that appear in the macroscopic equations as a function of the angle that the torus axis forms with the $z$-axis. The torus modulates the $D_{11}$ and $D_{33}$ components (top panel of figure 6) and produces a weaker modulation of $D_{22}$ (not shown). When the axis of the torus is not aligned with either the $x$ or $z$ axis the components $D_{13}$ and $D_{31}$ are equal, small in magnitude and negative. As the average alignment on the surface of the torus is non-zero, the anisotropy field $\boldsymbol{q}$ defined in eq. (29) is non-zero and its component vary with the orientation of the torus axis.

\section{CONCLUSIONS}

$\mathcal{Q}$-tensor codes are a powerful tool to determine the alignment of liquid crystals in complex geometries and the response of liquid crystals under time dependent fields. The drawback of this flexibility is that the alignment equations are stiff and, hence, computationally expensive. Over the last five years our group has been developing fast $\mathcal{Q}$-tensor codes that provide accurate alignment estimates in the absence of defects. Two of the algorithms that we have developed, ${ }^{7}{ }^{11}$ summarized in sections 2 and 3, concern pure liquid crystals and are based on a multiple-scale expansion of the $\mathcal{Q}$-tensor equation. The 


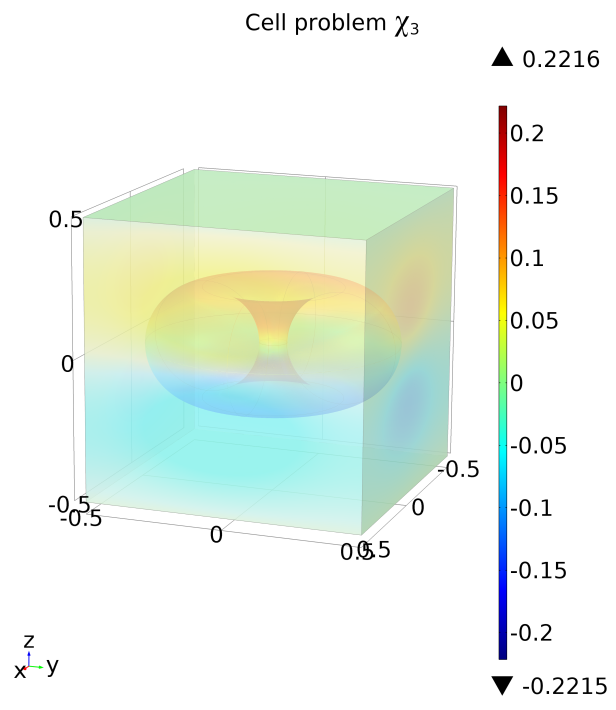

Figure 5. Numerical solution of the elastic cell problem for $\chi_{3}$ for a toroidal inclusion. In addition to determining the elasticity tensor, $\chi_{k}$ partly determines the leading order correction to the alignment $\boldsymbol{a}_{1}=\sum_{k=1}^{3} \chi_{k} \frac{\partial \boldsymbol{a}_{0}}{\partial x_{k}}$. Note that equations (32) only define $\chi_{k}$ up to a constant.
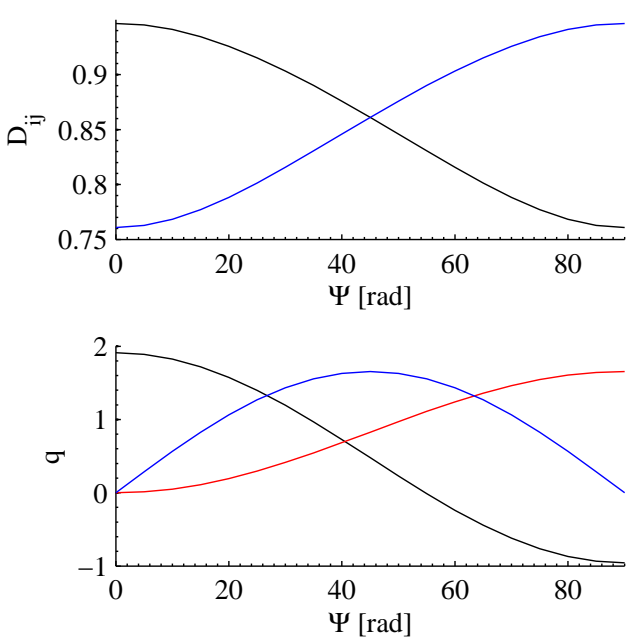

Figure 6. Components of elasticity tensor $D_{i j}$, eq. (32), ( $D_{11}$ black, $D_{33}$ blue) and forcing term $q_{i}$, eq. (33), $\left(q_{1}\right.$ black, $q_{2}$ red and $q_{4}$ blue) computed for a toroidal inclusion with axis in the $(x, z)$-plane forming an angle $\Psi$ with respect to the $z$-axis. The components $q_{3}$ and $q_{5}$ are zero as the torus does not induce out of plane twist.

third one, ${ }^{29}$ summarized in section 4 , describes the alignment of a liquid crystal with fixed inclusions (e.g. nanopillars) and is based around the application of homogenization theory to the $\mathcal{Q}$-tensor equations.

These seemingly disparate codes have, however, a common thread: in the absence of defects, the liquid crystal free energy is dominated by its thermotropic part. To first approximation, the $\mathcal{Q}$-tensor lies on the equilibrium surface of the thermotropic free energy. The net effect of elastic and electrostatic forces is to move the $\mathcal{Q}$-tensor along this surface. Therefore, the re-orientation dynamics of the liquid crystal, which is captured by all the algorithms described in this paper, is (i) low dimensional and (ii) with a single time scale. The existence of the invariant manifold is self-evident in the DFQTA and DFQTAF methods described in sections 2 and 3 for pure liquid crystals. It is more hidden in the homogenization method used for doped liquid crystal summarized in section 4 . In this case, the existence of the invariant manifold manifests itself in the fact that the macroscopic alignment field described by equations (27b) does not depend explicitly on the spatial coordinates at the microscopic (cell) level. The added benefit of using homogenization to obtain the macroscopic equations for a doped liquid crystal is that not only the equations are non-stiff, but that we have a clear and algorithmic connection between the microscopic properties of the liquid crystal and the control parameters in the macroscopic equations. These observations lead naturally to a series of questions that we are currently trying to address. The first, is what can be done if defects are present. We are currently working at incorporating the treatment of defects in these algorithms, starting from the fairly basic approach of using numerical patching between the defect and defect-free regions. A second one is whether the homogenization approach can be extended to rotating and translating particles. Works along these lines have been considered, for example, in the context of rigid body rotation of porous structures. ${ }^{31}$

It is clear, though, that, already at this early stage, the many orders of magnitude increase in computational speed that the algorithms outlined in this paper offer, opens the way to the realistic simulation and optimization of liquid crystal devices that operate in the absence of defects.

\section{ACKNOWLEDGMENTS}

We would like to thank our colleagues Malgosia Kaczmarek and Tim Sluckin for many helpful discussions. This work has been partly supported by the EPSRC grant EP/J006920/1. The authors acknowledge the use of the IRIDIS High 
Performance Computing Facility, and associated support services at the University of Southampton, in the completion of this work.

\section{REFERENCES}

[1] M. Kaczmarek, A. Dyadyusha, S. Slussarenko, and I. C. Khoo, "The role of surface charge field in two-beam coupling in liquid crystal cells with photoconducting polymer layers," J. Appl. Phys. 96, pp. 2616-2623, Sept. 2004.

[2] A. d'Alessandro, B. Bellini, D. Donisi, R. Beccherelli, and R. Asquini, "Nematic Liquid Crystal Optical Channel Waveguides on Silicon," IEEE J. Quant. Elect. 42(10), pp. 1084-1090, 2006.

[3] J. Beeckman, K. Neyts, and P. J. M. Vanbrabant, "Liquid-crystal photonic applications," Opt. Eng. 50(8), p. 081202, 2011.

[4] F. S. Y. Yeung and H. S. Kwok, "Truly bistable twisted nematic liquid crystal display using photoalignment technology," Applied Physics Letters 83(21), pp. 4291-4293, 2003.

[5] I. W. Stewart, The static and dynamic continuum theory of liquid crystals: a mathematical introduction, Taylor \& Francis, 2004.

[6] A. Majumdar and A. Zarnescu, "Landau-de gennes theory of nematic liquid crystals: the oseen-frank limit and beyond," Arch. Rat. Mech. Anal. 196(1), pp. 227-280, 2010.

[7] K. R. Daly, G. D’Alessandro, and M. Kaczmarek, "An Efficient $\mathcal{Q}$-Tensor-Based Algorithm for Liquid Crystal Alignment away from Defects," SIAM J. Appl. Math. 70(8), pp. 2844-2860, 2010.

[8] A. Sonnet, A. Kilian, and S. Hess, "Alignement tensor versus director: Description of defects in nematic liquid crystals," Phys. Rev. E 52(1), pp. 718-722, 1995.

[9] R. James, E. Willman, A. Fernandez, and S. Day, "Computer modeling of liquid crystal hydrodynamics," Magnetics, IEEE Transactions on 44, pp. 814-817, June 2008.

[10] M. G. Clark and F. M. Leslie, "A calculation of orientational relaxation in nematic liquid crystals," Proc. R. Soc. Lond. A 361(1707), pp. 463-485, 1978.

[11] Y. Murugesan, G. de Matteis, and G. D'Alessandro, "A fast $\mathcal{Q}$-tensor algorithm with flow," To be submitted to Phys Rev E, 2014.

[12] A. Sonnet, P. Maffettone, and E. Virga, "Continuum theory for nematic liquid crystals with tensorial order," J. NonNewtonian Fluid Mech. 119, pp. 51 - 59, 2004.

[13] L. M. Lopatina and J. V. Selinger, "Theory of Ferroelectric Nanoparticles in Nematic Liquid Crystals," Phys. Rev. Lett. 102, p. 197802, May 2009.

[14] L. M. Lopatina and J. V. Selinger, "Maier-Saupe-type theory of ferroelectric nanoparticles in nematic liquid crystals," Phys. Rev. E 84, p. 041703, Oct 2011.

[15] F. Brochard and P. G. de Gennes, "Theory of magnetic suspensions in liquid crystals," J. Phys, France 31, pp. 691708, July 1970.

[16] S. V. Burylov and Y. L. Raikher, "Macroscopic Properties of Ferronematics Caused by Orientational Interactions on the Particle Surfaces. I. Extended Continuum Model,” Mol. Cryst. Liq. Cryst. 258, pp. 107-122, 1995.

[17] S. V. Burylov and Y. L. Raikher, "Macroscopic Properties of Ferronematics Caused by Orientational Interactions on the Particle Surfaces. II. Behavior of Real Ferronematics in External Fields," Mol. Cryst. Liq. Cryst. 258, pp. 123-141, 1995.

[18] N. Podoliak, O. Buchnev, O. Buluy, G. D’Alessandro, M. Kaczmarek, Y. Reznikov, and T. J. Sluckin, "Macroscopic optical effects in low concentration ferronematics," Soft Matter 7(10), pp. 4742-4749, 2011.

[19] A. Mertelj, D. Lisjak, M. Drofenik, and M. Čopič, "Ferromagnetism in suspensions of magnetic platelets in liquid crystal," Nature 504, pp. 237-241, Dec. 2013.

[20] B. Senyuk, Q. Liu, S. He, R. D. Kamien, R. B. Kusner, T. C. Lubensky, and I. I. Smalyukh, "Topological colloids," Nature 493, pp. 200-205, Jan. 2013.

[21] S. Čopar, M. R. Dennis, R. D. Kamien, and S. Žumer, "Singular values, nematic disclinations, and emergent biaxiality," Phys. Rev. E 87, p. 050504, May 2013.

[22] T. Hegmann, H. Qi, B. Kinkead, V. Marx, H. Girgis, and P. Heiney, "Functionalized metal and semiconductor nanaporticle doped liquid crystals - Modifying optical and electro-optical properties," Can. Metallurgical Quat. 48(1), pp. $1-10,2009$. 
[23] S. K. Prasad, K. L. Sandhya, G. G. Nair, U. S. Hiremath, C. V. Yelamaggad, and S. Sampath, "Electrical conductivity and dielectric constant measurements of liquid crystal-gold nanoparticle composites," Liq. Cryst. 33(10), pp. 1121$1125,2006$.

[24] H. Qi, B. Kinkead, and T. Hegmann, "Unprecedented Dual Alignment Mode and Freedericksz Transition in Planar Nematic Liquid Crystal Cells Doped with Gold Nanoclusters," Adv. Funct. Mater. 18(2), pp. 212-221, 2008.

[25] M. Urbanski, B. Kinkead, H. Qi, T. Hegmann, and H.-S. Kitzerow, "Electroconvection in nematic liquid crystals via nanoparticle doping," Nanoscale 2(7), pp. 1118-1121, 2010.

[26] D. F. Gardner, J. S. Evans, and I. I. Smalyukh, "Towards reconfigurable optical metamaterials: Colloidal nanoparticle self-assembly and self-alignment in liquid crystals," Mol. Cryst. Liq. Cryst. 545(1), pp. 3/[1227]-21/[1245], 2011.

[27] B. Senyuk, J. S. Evans, P. J. Ackerman, T. Lee, P. Manna, L. Vigderman, E. R. Zubarev, J. v. d. Lagemaat, and I. I. Smalyukh, "Shape-dependent oriented trapping and scaffolding of plasmonic nanoparticles by topological defects for self-assembly of colloidal dimers in liquid crystals," Nano Lett. 12(2), pp. 955-963, 2012.

[28] O. Buchnev, J. Y. Ou, M. Kaczmarek, N. I. Zheludev, and V. A. Fedotov, "Electro-optical control in a plasmonic metamaterial hybridised with a liquid-crystal cell," Opt. Express 21, pp. 1633-1638, Jan 2013.

[29] T. Bennett, K. Daly, and G. D'Alessandro, "Multiscale models of colloidal dispersion of nanoparticles in nematic liquid crystals," Submitted to Phys. Rev. E, 2014.

[30] G. Pavliotis and A. Stuart, Multiscale methods: averaging and homogenization, Springer-Verlag, New York, 1 ed., 2007.

[31] O. Iliev, A. Mikelic, and P. Popov, "On upscaling certain flows in deformable porous media," Multiscale Model $\operatorname{Sim}$ 7(1), pp. 93-123, 2008. 